\title{
The Language of Caring: Quantitating Medical Practice Patterns using Symbolic Dynamics
}

\author{
J. Paladino ${ }^{1}$, A. M. Kaynar ${ }^{2}$, P. S. Crooke ${ }^{3}$, and J. R. Hotchkiss ${ }^{4}$ * \\ ${ }^{1}$ Department of Medicine, University of Hawaii \\ ${ }^{2}$ Departments of Critical Care Medicine and Anesthesiology, University of Pittsburgh \\ ${ }^{3}$ Department of Mathematics, Vanderbilt University \\ ${ }^{4}$ Departments of Critical Care Medicine and Medicine, \\ University of Pittsburgh and Pittsburgh Veterans Affairs Healthcare System
}

\begin{abstract}
Real-world medical decisions rarely involve binary sole condition present or absentpatterns of patient pathophysiology. Similarly, provider interventions are rarely unitary in nature: the clinician often undertakes multiple interventions simultaneously. Conventional approaches towards complex physiologic derangements and their associated management focus on the frequencies of joint appearances, treating the individual derangements of physiology or elements of intervention as conceptually isolated. This framework is ill suited to capture either the integrated patterns of derangement displayed by a particular patient or the integrated patterns of provider intervention. Here we illustrate the application of a different approach-that of symbolic dynamics-in which the integrated pattern of each patients derangement, and the associated provider response, are captured by defining words based on the elements of the pattern of failure. We will use as an example provider practices in the context of mechanical ventilation- a common, potentially harmful, and complex life support technology. We also delineate other domains in which symbolic dynamics approaches might aid in quantitating practice patterns, assessing quality of care, and identifying best practices.
\end{abstract}

Key words: symbol dynamics, patient care, language AMS subject classification: $92 \mathrm{C} 60$

\footnotetext{
${ }^{*}$ Corresponding author. E-mail: hotchkissjr@upmc.edu
} 


\section{Introduction}

Real-world medical decisions rarely involve binary sole condition present or absent- patterns of patient pathophysiology. Rather, the health care provider generally confronts a set of derangements, each of which may interact with (and therefore must be considered in the context of) many of the other abnormalities. For example, a patient undergoing mechanical ventilation might have a high airspace distending pressure, a low blood pressure, and a high blood carbon dioxide content. Similarly, a patient with chronic kidney disease might have high serum calcium, high serum phosphorus, and low serum parathyroid hormone levels as the result of treatment. In both cases, management decisions that address only one of the derangements could actually cause harm.

Similarly, provider interventions are rarely unitary in nature: the clinician often undertakes multiple interventions simultaneously. For example, in the aforementioned mechanical ventilation example, a provider might decrease the size of the patients breaths, increase the rate at which breaths are provided, and administer fluid. Conversely, in the patient with kidney disease, a provider might decrease a medication that raises calcium while increasing a medication that binds phosphorus in the gut. In each case, interventions that are plausible but address only one aspect of the patients derangement would be likely to cause some degree of harm.

Conventional approaches towards complex physiologic derangements and their associated management capture the frequencies of joint appearances, treating the individual derangements of physiology or elements of intervention as conceptually isolated. This framework is inherently population-focused rather than patient-specific. Such an approach is ill suited to capture the integrated patterns of derangement displayed by a particular patient (patterns of failure), as well as the integrated patterns of provider intervention. Here we will illustrate the application of a different approach- that of symbolic dynamics- in which the integrated pattern of the patients derangement is captured by defining words based on the elements of the pattern of failure. The interventions undertaken by the provider are similarly characterized. Combinatorial analyses can then be conducted utilizing the individual or joint appearance frequency of such words. We will use data from an ongoing study of provider practice patterns during mechanical ventilation to illustrate these concepts.

\section{Encoding Interventions and Outcomes}

Symbolic dynamics approaches have been applied to complex dynamic systems ranging from disturbances of cardiac rhythm and integrated neurological function to astrophysics and internal combustion engines ([1, 2, 3, 4, 5, 6, 7]). In our approach, disparate streams of physiologic data (such as peak and plateau airspace pressures, or calcium and phosphorus levels) are evaluated at sequential time points. Each data stream is converted into a sequence of symbols. For each physiologic element, the patient values at each time point are defined as being acceptable, too high, or too low. For each possible provider intervention, the symbol is defined based on the relationship between the current value and that of the preceding time point:

Patient Outcome Values 
value acceptable $\longrightarrow$ outcome symbol $=A$

value too high $\longrightarrow$ outcome symbol $=B$

value too low $\longrightarrow$ outcome symbol $=C$

$\underline{\text { Provider Interventions }}$

no change $\longrightarrow$ intervention symbol $=W$

increase dose $\longrightarrow$ intervention symbol $=X$

decrease dose $\longrightarrow$ intervention symbol $=Y$

More highly nuanced encodings (such as quintiles with very high, high, acceptable, low, very low) are readily incorporated. These symbols can be concatenated into words that describe the care process at each point in time.

\begin{tabular}{l|l|l|l} 
Phenomenon & Point One & Point Two & Point Three \\
Patient Physiologic Status & $B A C B$ & $A A C B$ & $A A A B$ \\
Provider Response & $W X Y Y$ & $X W X Y$ & $W W X Y$
\end{tabular}

The care process is thereby encoded as 2 sequences of words that describe the patients physiologic status and the associated provider responses.

We are using a non-linear digital simulator of cardiorespiratory physiology during mechanical ventilation to explore expert patterns of practice when confronting different, clinically relevant (simulated) patient pathophysiologies $([8,9,10,11,12,13,14,15])$. Users are confronted with a series of 100 standardized, dynamically responsive patients suffering a variety of physiologic derangements, and must attempt to attain pre-specified physiologic goals for each patient by adjusting ventilator settings germane to volume cycled ventilation. In the simplest combinatorial formulation, there are 24 modes of failure (such as hypoxia combined with hypotension and respiratory acidosis), and a universe of 1,458 possible qualitative ventilator changes (response patterns). At each point, the simulator records the value of each outcome of interest and the changes in ventilator settings selected by the user. This allows construction of appearance frequency tables for each mode of failure and each pattern of provider response. The left panel depicts the failure patterns encountered by expert practitioners, and the right panel is a frequency histogram of the ensembles of ventilator changes made by the same set of experts.

The frequency with which a single provider or a panel of experts responds to a particular pattern of failure by implementing a specific intervention pattern can be determined. Simple counting algorithms can be used to define a response matrix in which the matrix elements represent the frequency with which a given pattern is followed by a particular response (only the percent frequencies of the first 10 response patterns are shown):

This approach fundamentally represents the purely combinatorial translation of patient characteristics and provider responses into a quantitative language. Accordingly, once the primitive elements of the language (patient characteristics and provider responses of interest) are defined, the analysis is objective, unbiased, and not dependent on assumptions regarding the statistical distributions underlying the relevant elements or their associations. The outputs (response matrices) are highly amenable to application of contemporary pattern recognition techniques, such as calcu- 

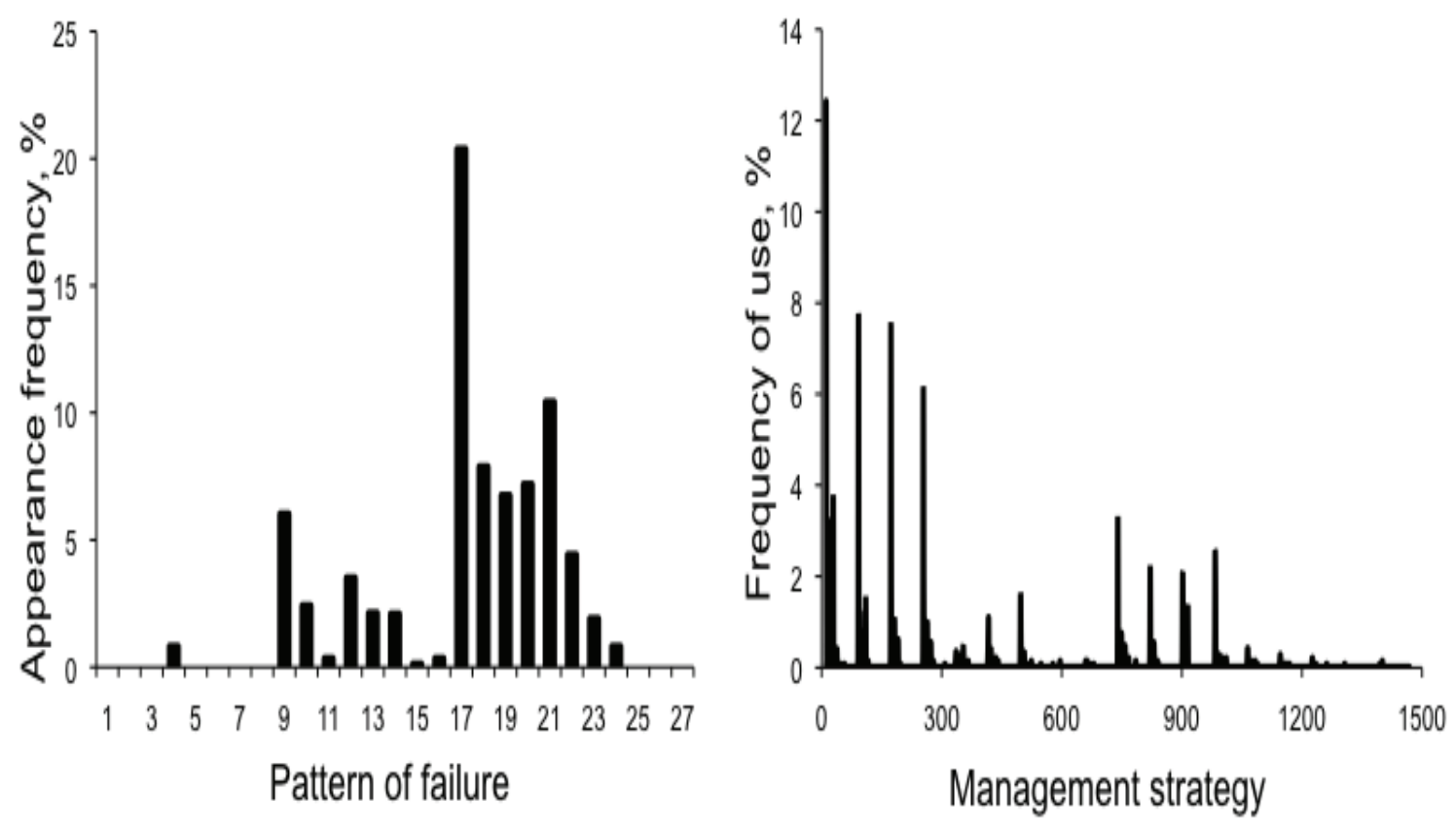

Figure 1: Appearance frequency histograms for failure patterns and intervention patterns.

\section{Failure pattern $\Rightarrow$}

\begin{tabular}{|c|c|c|c|c|c|c|c|c|c|c|c|c|c|c|c|c|c|c|c|c|c|c|c|c|}
\hline & 1 & 2 & 3 & 4 & 5 & 6 & & 8 & 9 & 10 & 11 & 12 & 13 & 14 & 15 & 16 & & & & & & & & \\
\hline 1111111 & 0 & 0 & 0 & 0 & 0 & 0 & & 0 & 4.1 & 0 & 13 & 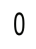 & 8.3 & 3.6 & 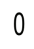 & 0 & & & & & & & & 5.3 \\
\hline 1111112 & 0 & 0 & 0 & 0 & 0 & 0 & & 0 & 1.4 & 0 & 0 & 0 & 5.6 & 3.6 & ( & & & & & & & & & 16 \\
\hline 111113 & 0 & 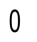 & 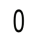 & 0 & 0 & 0 & & 0 & 0 & 0 & & 0 & 0 & 0 & U & & & & & & & & & 0 \\
\hline 1111121 & 0 & 0 & 0 & 0 & 0 & 0 & & 0 & 0 & 0 & & & 0 & 0 & & & & & & & & & & 0 \\
\hline 1111122 & 0 & 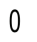 & 0 & 0 & 0 & 0 & & & 0 & 0 & & & 0 & 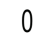 & & & & & & & & & & U \\
\hline 1111123 & 0 & 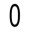 & 0 & 0 & 0 & 0 & & 0 & 0 & 0 & & 0 & 0 & 0 & & & & & & & & & & 0 \\
\hline 1111131 & 0 & 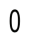 & 0 & 0 & 0 & 0 & & 0 & 0 & 0 & & & 0 & 0 & & & & & & & & & & 0 \\
\hline 1111132 & 0 & c & 0 & 0 & 0 & 0 & & ( & 0 & 0 & & & 0 & & & & & & & & & & & 0 \\
\hline 1111133 & 0 & 0 & 0 & 0 & 0 & 0 & & 0 & 0 & 0 & & & 0 & 0 & & & & & & & & & & 0 \\
\hline 1111211 & 0 & 0 & . & 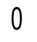 & 0 & 0 & & 0 & 7.5 & 0 & & . & 0 & 3.6 & & & & & & & & & & 2.6 \\
\hline$*$ & * & * & * & * & * & * & & * & * & * & * & * & * & * & & & & & & & & & & * \\
\hline * & * & * & * & * & * & * & & * & * & $\pi$ & * & * & * & $x$ & & & & & & & & & & * \\
\hline & * & & & ${ }^{*}$ & & & & & * & & & & 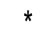 & & & & & & & & & & & \\
\hline
\end{tabular}

Figure 2: Matrix of expert panel response frequencies for different failure patterns. 


$\begin{array}{cccccccccccc} & \text { CON } & 1 & 2 & 3 & 4 & 5 & 6 & 7 & 8 & 9 & 10 \\ \text { CON } & -5.69 & -1.43 & -2.65 & 0.097 & -2.32 & -0.73 & -0.47 & -2.61 & -1.85 & -0.76 & -2.13 \\ 1 & -1.43 & -5.69 & -0.5 & 1.512 & -0.63 & -0.31 & 0.78 & -0.72 & -0.45 & 0.153 & 0.055 \\ 2 & -2.65 & -0.5 & -5.69 & 0.571 & -1.22 & 0.43 & -0.03 & -2.05 & -1.01 & -0.07 & -0.41 \\ 3 & 0.097 & 1.512 & 0.571 & -5.69 & 0.857 & 2.293 & 2.028 & 0.712 & 0.86 & 2.09 & 1.415 \\ 4 & -2.32 & -0.63 & -1.22 & 0.857 & -5.69 & 0.242 & -0.27 & -2.01 & -1.09 & 0.017 & -1.12 \\ 5 & -0.73 & -0.31 & 0.43 & 2.293 & 0.242 & -5.69 & 1.483 & 0.183 & 0.232 & 1.101 & 0.281 \\ 6 & -0.47 & 0.78 & -0.03 & 2.028 & -0.27 & 1.483 & -5.69 & -0.44 & 0.368 & 1.047 & 0.737 \\ 7 & -2.61 & -0.72 & -2.05 & 0.712 & -2.01 & 0.183 & -0.44 & -5.69 & -1.32 & -0.35 & -0.65 \\ 8 & -1.85 & -0.45 & -1.01 & 0.86 & -1.09 & 0.232 & 0.368 & -1.32 & -5.69 & 0.396 & -0.31 \\ 9 & -0.76 & 0.153 & -0.07 & 2.09 & 0.017 & 1.101 & 1.047 & -0.35 & 0.396 & -5.69 & 0.413 \\ 10 & -2.13 & 0.055 & -0.41 & 1.415 & -1.12 & 0.281 & 0.737 & -0.65 & -0.31 & 0.413 & -5.69\end{array}$

Figure 3: z-scores for differences between providers and as compared to consensus practice pattern.

lation of difference matrices (vide infra) and clustering algorithms.

\section{Analysis Symbol Strings}

In the style of Tang and Daw, the difference between the response patterns of a particular practitioner and either the consensus pattern (matrix defined on a panel of experts) or the pattern adopted by a particular practitioner can be computed. Defining $f_{i j}^{(k)}$ as the frequency with which a particular practitioner $(k)$ (or set of practitioners) responds to a pattern of derangement $(i)$ by implementing a pattern of changes $(j)$, the elements of the difference matrix are given by:

$$
\Delta_{r, s}=\sqrt{\sum_{i=1}^{n} \sum_{j=1}^{n}\left(f_{i j}^{(r)}-f_{i j}^{(s)}\right)^{2}}
$$

And a difference matrix describing the distances between each pair of users, as well as the distance between each user and the expert consensus profile $(C O N)$, can be constructed. If simple descriptive statistics are computed on the population of users, these distances can be expressed as the commonly used $z$-scores:

$$
z_{r, s}=\frac{\Delta_{r, s}-\mu_{p o p}}{\sigma_{p o p}}
$$

where $\mu_{p o p}$ is the mean of the differences in the population and $\sigma_{p o p}$ is its standard deviation. For 8 individuals in the aforementioned study, the symmetric $z$-score matrix is show in Figure 3.

The ability to objectively quantitate the patterns of failure in a given patient, and the patterns of intervention with which providers respond to specific patterns of patient derangement, has potential 
applicability both for assessing provider performance and in the construction of expert decision support systems.

For example, data such as those we present could be used to assess the cognitive performance of providers in the domain of mechanical ventilation. At present, there is no good method for assessing practitioner competence in managing this common, lifesaving, but potentially injurious cornerstone of modern intensive care medicine. Application of simulation based technology, coupled with symbolic dynamics approaches to provider responses, could allow rigorous comparison between a given providers intervention patterns and those implemented by experts confronted with the same standardized problems. Simulation based technology allows the presentation of a large number of standardized patient problems that are similar to those encountered in clinical practice, and resembles clinical practice in that the participant is free to choose any ensemble of interventions, and must respond to the consequences of each set of choices. Use of symbolic dynamics assessment tools allows rigorous quantitation of provider practice patterns and comparison to expert response patterns.

Similarly, symbolic dynamics approaches can be applied to characterize provider practice patterns in settings where there are extensive and relatively uniform patient data and provider management changes are clearly documented. These requirements are satisfied in numerous clinical settings, including the management of calcium, phosphorus, and parathyroid hormone, and the management of anemia, in the End Stage Renal Disease population. Symbolic dynamics approaches can be used to quantify variability in provider practice, identify expert or consensus practice patterns, and quantitate the extent to which a given provider responds to specific patient failure patterns in a fashion similar to experts.

Finally, the capacity to objectively and systematically quantitate patterns of derangement and patterns of response can be coupled with data mining techniques to identify potential best practices at the level of individual patients. In the first approach, the response matrix of an expert panel can be coupled to a look-up algorithm, so that for a given patient pattern the user is presented with the frequencies with which a panel of experts implemented each possible response pattern. This approach effectively and in an unbiased fashion distills expert opinion into objective data.

In a second approach, given an adequate volume of sequential data, those patterns of response most frequently attaining therapeutic goals can be identified by tracking the effects of particular intervention patterns in the setting of specific patient derangements. The following terms for each patient at each point examined are defined:

- $S(n)=1 \longrightarrow$ all outcomes for that patient are satisfactory at time point $n$

- $S(n)=0 \longrightarrow$ at least one outcome for that patient is unsatisfactory at time point $n$

Furthermore, we can define the dynamic patterns:

- PatientPattern $(n)=$ the symbolic pattern of patient physiology at time point $n$

- InterventionPattern $(n)=$ the symbolic pattern of provider interventions at time point $n$. 
A response matrix of the form (patient derangement) $\mathrm{x}$ (response pattern) can be constructed, where the elements $(i, j)$ reflect the probability with which response pattern $(j)$, in the setting of derangement $(i)$, leads to satisfactory resolution of the patients derangement, based on the subsequent pattern in that patient. In particular, the $(i, j)$ element is the probability that $S(n+1)=1$ and $S(n)=0$ for a given PatientPattern $(n)$ and InterventionPattern(n). Those interventions most likely to correct a given ensemble of derangements, and their population averaged likelihood of success, can be identified, tabulated, and presented. Notably, because a symbolic dynamics approach explicitly addresses the complete set of derangements prevailing in each patient and the associated responses to specific intervention patterns (rather than population averages or medians), these approaches are de facto consistent with individualized medicine:

Patients with this particular pattern of derangements display satisfactory resolution of their disorders when treated with the following patterns of intervention ...

\section{Conclusion}

In summary, there is growing interest in simulation based approaches to medical education as well as in data mining using large clinical databases to identify putative best practices. Symbolic dynamics approaches to characterizing patterns of patient derangement and provider response at the level of individual patients may prove valuable tools both for gauging provider competence and performance, as well as for identifying optimal approaches to patient management in a manner commensurate with individualized medicine.

\section{Acknowledgements}

This research was partially supported by the National Institute of Health: R01HL084113 and T32HL007820.

\section{References}

[1] R. Engbert, C. Scheffczyk, R.T. Krampe, M. Rosenblum, J. Kurths, R. Kliegl. Tempo-induced transitions in polyrhythmic hand movements. Phys. Rev. E, 56(1997), No. 5, 5823-5833.

[2] B. Hao. Symbolic dynamics and characterization of complexity. Physica D, 51(1991),161176.

[3] R. Engbert, M. Schiek, J. Kurths, R. Krampe, R. Kliegl, F. Drepper. Symbolic dynamics of physiological synchronization: Examples from bimanual movements and cardiorespiratory interaction. Nonlin. Anal. Theor. Meth. Appl., 30(1997), No. 2, 973-984. 
[4] U. Schwarz, A.O. Benz, J. Kurths, A. Witt. Analysis of solar spike events by means of symbolic dynamics methods. Astron. Astrophys., 277(1993), 215-224.

[5] X.Z. Tang, E.R. Tracy, A.D. Boozer, A. Debrauwa, R. Brown. Symbol sequence statistics in noisy chaotic signal reconstruction. Phys. Rev. E, 51(1995), 3871-3889.

[6] C.S. Daw, M.B. Kennel, C.E.A. Finney, F.T. Connolly. Observing and modeling nonlinear dynamics in an internal combustion engine. Phys. Rev. E, 57(1998), No. 3, 2811-2819.

[7] C.S. Daw, C.E.A. Finney, E.R. Tracy. A review of symbolic analysis of experimental data. Rev. Scien. Instru., 47(2003), No. 2, 915-930.

[8] J.R. Hotchkiss, P.S. Crooke, A.B. Adams, J.J. Marini. Implications of a biphasic two compartment model of constant flow ventilation for the clinical setting. J. Crit. Care. 9(1994), No. 2, 114-123.

[9] P.S. Crooke, J.D. Head, J.J. Marini, J.R. Hotchkiss. Patient-ventilator interaction: A general model for non-passive mechanical ventilation. IMA J. Math. Appl. Med. Biol., 15(1998), 321-337.

[10] P.S. Crooke, S. Hota, J.J. Marini, J.R. Hotchkiss. A mathematical model for carbon dioxide exchange during mechanical ventilation with TGI. Math. Comp. Mod., 29(1999), 45-61.

[11] A.B. Adams, P. Bliss, J.R. Hotchkiss. Effects of respiratory impedance on the performance of bi-level pressure ventilators. Respir. Care, 45(2000), No. 4, 390-400.

[12] J.R. Hotchkiss, D.J. Dries, J.J. Marini, P.S. Crooke. Dynamical behavior during noninvasive ventilation: chaotic support? Am. J. Resp. Crit. Care Med., 163(2001), 374-378.

[13] J.R. Hotchkiss, A.B. Adams, M.K. Stone, D.J. Dries, J.J. Marini. Oscillations and noise: inherent instability of pressure support ventilation? Am. J. Resp. Crit. Care Med., 165(2002), $47-53$.

[14] P.S. Crooke, J.R. Hotchkiss, J.J. Marini. Linear and nonlinear mathematical models for noninvasive, passive ventilation. Math. Comp. Mod., 35(2002),1297-1313.

[15] P.S. Crooke, J.R. Hotchkiss, J.J. Marini. Modeling recruitment maneuvers with a variable compliance model for pressure preset ventilation. J. Theor. Med., 43(2002),No. 3, 197-207. 